\title{
Comparison of Program Resources Required for Colonoscopy and Fecal Screening: Findings From 5 Years of the Colorectal Cancer Control Program
}

\author{
Sujha Subramanian, $\mathrm{PhD}^{1}$; Florence K.L. Tangka, $\mathrm{PhD}^{2}$; Sonja Hoover, MPP${ }^{1}$; Maggie Cole-Beebe, $\mathrm{PhD}^{1}$; \\ Djenaba Joseph, MD, MPH${ }^{2}$; Amy DeGroff, $\mathrm{PhD}, \mathrm{MPH}^{2}$
}

\begin{abstract}
Accessible Version: www.cdc.gov/pcd/issues/2019/18_0338.htm
Suggested citation for this article: Subramanian S, Tangka FK, Hoover S, Cole-Beebe M, Joseph D, DeGroff A. Comparison of Program Resources Required for Colonoscopy and Fecal Screening: Findings From 5 Years of the Colorectal Cancer Control Program. Prev Chronic Dis 2019;16:180338. DOI: https:// doi.org/10.5888/pcd16.180338.
\end{abstract}

\section{PEER REVIEWED}

\section{Summary}

What is already known on this topic?

Three years of program data from the Centers for Disease Control and Prevention's (CDC's) Colorectal Cancer Control Program (CRCCP) showed that although the clinical cost of colonoscopy programs was higher than the clinical cost for guaiac fecal occult blood tests and fecal immunochemical tests programs, the cost of nonclinical services required to manage the programs and deliver the screenings was similar.

What is added by this report?

CDC and RTI International collected 5 years of cost data from 29 CRCCP grantees by using a standardized data collection instrument and assessed differences in costs by screening test used.

What are the implications for public health practice?

CRCCP grantees incurred costs in addition to the clinical cost of the screening procedures to support planning and management, contracting with providers, and tracking patients.

\section{Abstract}

\section{Introduction}

Colonoscopy and guaiac fecal occult blood tests and fecal immunochemical tests (FOBT/FIT) are the most common colorectal cancer screening methods in the United States. However, information is limited on the program resources required over time to use these tests.

\section{Methods}

We collected cost data from 29 Centers for Disease Control and Prevention Colorectal Cancer Control Program (CRCCP) grantees by using a standardized data collection instrument for 5 program years (2009-2014). We created a panel data set with 124 records and assessed differences by screening test used.

\section{Results}

Forty-four percent of all programs $(\mathrm{N}=124)$ offered colonoscopy (55 of 124), 32\% (39 of 124) offered FOBT/FIT, and 24\% (30 of $124)$ offered both. Overall, total cost per person was higher in program year $1(\$ 3,962)$, the beginning of CRCCP than in subsequent program years $(\$ 1,714)$. The cost per person was $\$ 3,153$ for programs using colonoscopy and $\$ 1,291$ for those using FOBT/FIT with diagnostic colonoscopy. The average clinical cost per person was $\$ 1,369$ for colonoscopy and $\$ 280$ for FOBT/FIT during the program (these do not reflect cost of repeated FOBT/ FIT screens). Programs serving a large number of people had lower per-person costs than those serving a small volume, probably because of fixed costs related to nonclinical expenses.

\section{Conclusion}

Colorectal cancer screening programs incur costs in addition to the clinical cost of the screening procedures to support planning and management, contracting with providers, and tracking patients. Because programs can achieve potential economies of scale, partnerships among smaller programs for screening delivery could decrease overall costs.

\section{Introduction}

The Centers for Disease Control and Prevention (CDC) initiated the Colorectal Cancer Control Program (CRCCP) in 2009 to promote and provide screening to increase colorectal cancer (CRC) screening uptake in target populations. Under the program, CDC funded 29 grantees (25 states and 4 tribal organizations); grantees 
generally offered free screening colonoscopy or fecal tests to lowincome people who were uninsured or underinsured. In an interim analysis of CRCCP, we assessed differences in costs of clinical and nonclinical screening incurred by CRCCP grantees during the first 3 years of the program and found that the cost of screening and diagnostic services per person served was $\$ 1,150$ for colonoscopy programs and $\$ 304$ for FIT/FOBT-based programs (1). Overall, FOBT/FIT-based programs and colonoscopy programs incurred substantial nonclinical costs per person served $(\$ 1,018$ for colonoscopy and $\$ 980$ for FIT/FOBT). Examples of nonclinical costs were managing contracts with providers and program management. These findings indicated that although the clinical cost of colonoscopy programs was higher than the clinical cost of FOBT/FIT programs, the cost of nonclinical services required to manage the programs and deliver the screenings was similar.

Our study expands on this prior analysis by evaluating cost over the 5-year period of the program and potential economies of scale in program implementation by assessing factors affecting the cost of screening provision. The large sample size available for analysis allowed us to perform multivariate analysis to evaluate the effect of large versus small programs on clinical and nonclinical costs, controlling for factors such as geographic location and type of screening test used. Prior research involving other screening programs indicated that these programs have high fixed costs (2-4). We theorized that programs that screen a large number of people may have a lower cost per-person than programs that screen a smaller number, which could have important implications for program planning and implementation.

\section{Methods}

\section{Data collection}

We developed a web-based cost assessment tool, the CRCCP Cost Assessment Tool (CRCCP-CAT), to collect information from CRCCP grantees on their program activities and expenditures. CRCCP-CAT is based on established methods for collecting cost data (5-8); a previously published article and a companion article in this collection describe the development and testing of CRCCPCAT $(9,10)$. For the CRCCP analyses, we collected data from each of the 29 CRCCP grantees. The grantees completed the webbased CAT annually, on the basis of program year, for a 5-year period beginning in July 2009 and ending in June 2014.

We collected cost information on the following: program funding source (CDC; other federal, nonfederal, state, or in-kind) and budget categories (staff salaries, contract expenditures, purchases of materials and equipment, and administration or overhead costs). Program staff members allocated these costs to screening activities, promotion activities, and overall program activities such as program management, partnership development, and administration. Promotion costs are discussed in a companion article in this collection (11). On the basis of the data provided in CRCCP-CAT, we allocated proportions of staff salary (based on number of hours and percentage of time worked) to specific activities. We then aggregated data on labor costs, nonlabor costs, and in-kind contributions for each activity for each grantee by year. Summaries of these data were sent to grantees annually for their review and approval.

The total sample size available for analysis was 124 program years over the 5-year period. We created a panel data set, which included each year of the program as 1 entry, and we reported our sample size in program years. Massachusetts (all years) and the Alaska Native Tribal Health Commission (all years) were excluded from the analyses because we were unable to disaggregate the clinical and nonclinical costs from contract payments in sufficient detail. Alabama, California, Iowa, New Mexico, and Oregon were all excluded in year 1 because they had not yet begun activities; Georgia, Michigan, and Nevada were not included in year 1 because they had not yet begun CRCCP. Georgia was also excluded from year 2 and Oregon from years 2 and 3 because their screening activities had not yet commenced during those years.

\section{Descriptive analyses}

We stratified the programs by type of screening test used: colonoscopy, FOBT/FIT, and programs that used both tests. Fecal tests that include FOBT and FIT were offered as screening tests, and colonoscopy was offered as screening and for follow-up diagnostic procedures. Programs with both tests offered both fecal tests and colonoscopy for colorectal cancer screening. Some programs also offered surveillance colonoscopies, and these were reported separately from screening colonoscopies.

We identified key characteristics of the program, including the region and number of people served by the program, which was categorized as large $(>500)$, medium $(235-500)$, and small $(<235)$ on the basis of the distribution of the underlying data. We also reported screening and diagnostic procedures for each type of program, including number of people who were screened or received surveillance colonoscopies, number of diagnostic procedures, and number of people identified with polyps. Use rates for the procedures were derived from information provided in CRCCP-CAT and from $\mathrm{CRC}$ clinical data elements that were collected from all programs by CDC (Office of Management and Budget [OMB] control no. 0920-0745).

We stratified cost information by the following activities: 1) direct clinical activities, such as provision of screening tests, diagnostic services, and surveillance procedures; 2) direct nonclinic-

The opinions expressed by authors contributing to this journal do not necessarily reflect the opinions of the U.S. Department of Health and Human Services, the Public Health Service, the Centers for Disease Control and Prevention, or the authors' affiliated institutions. 
al activities, such as managing provider contracts and billing systems and providing patient navigation and patient support services; and 3) indirect nonclinical overarching activities, such as program management and administration (Box).

\section{Box. Component Activities of the Colorectal Cancer Control Program, 2009-2014}

Direct clinical activities
Screening and diagnostic services
Surveillance procedures
Direct nonclinical activities
Provider contracts, billing systems, other billing procedures
Patient navigation and support
Labor costs for screening and diagnostic services (if reported)
Ensure cancer treatment
Other screening provision activities

Indirect nonclinical overarching activities (related to both screening promotion and screening procedures)

Program management

Quality assurance/professional development

Partnership development and maintenance

Clinical and cost data collection and tracking

Program monitoring and evaluation

Administration

Other activities

We calculated the cost per person aggregated across all program years and the cost for each program year to examine patterns across the 5-year period. We estimated adjusted costs (multivariate regression controlling for region, size of population served, and type of screening test) for total cost per person for direct clinical costs, direct nonclinical costs, and indirect costs. We estimated the average incremental effect on cost of each explanatory variable as the difference from one of the exponentiated coefficients and multiplying by the mean of the variable. Cost data were adjusted for regional differences by using the Bureau of Labor Statistics' Employment Cost Index.

\section{Multivariable regression specification}

We used multivariate analysis to assess the effect of volume of people screened on cost per person. We examined the total cost per person served by 3 cost components: total direct clinical cost, total direct nonclinical cost, and total indirect cost (12-14). Results of a Hausman test indicated that a fixed effects model was not appropriate for this panel data and that a mixed effects model should be used (15). We used a generalized linear model (GLM) with log link and specified a gamma distribution. We included data for years 2 to 5 in the regression estimation. We excluded year 1 because this was the start-up period, anticipating that costs for this year would differ from other program years.

GLM with log link allowed us to exponentiate the coefficient estimates without the need for a retransformation as is required when estimating a log-linear model. Regression results were tabulated in terms of the incremental effect on average cost. We used the Stata statistical package, version 14.0 (StataCorp LLC) to conduct all regression analyses and statistical tests of the model.

\section{Results}

Overall, 44.4\% (55 of 124) of the programs assessed used colonoscopy as the primary screening test; $31.5 \%(\mathrm{n}=39)$ used FOBT/ FIT, and $24.2 \%(\mathrm{n}=30)$ used both tests (Table 1$)$. Of the programs that offered colonoscopy as the primary screening test, the greatest percentage $(36.4 \% ; n=20)$ was in the Northeast, whereas of the 39 programs that offered FOBT/FIT as the primary screening test, most $(61.5 \% ; \mathrm{n}=24)$ were in the West. Forty-three percent (13 of 30 ) of the programs offering both tests were also located in the West. Programs offering FOBT/FIT and both types of tests were more likely to serve a large population (FOBT/FIT, $46.2 \%$ [18 of 39]; both tests, $63.3 \%$ [19 of 30]) than colonoscopy programs (10.9\% [6 of 55]). On average, grantees using both tests screened 2,152 people over the 5-year period, followed by grantees using FOBT/FIT (683 people)) and grantees using colonoscopy (254 people). We also assessed program testing method by program characteristics (Table 2).

Overall, total cost per person decreased from year $1(\$ 3,962)$ to year $5(\$ 1,841)$; average cost across years $2,3,4$, and 5 was $\$ 1,714$. On average, the cost per person was highest in year 1 for each component. For example, in year 1, direct clinical cost per person was $\$ 1,068$, decreasing in year 2 to $\$ 793$, and remaining similar over the remaining years (Figure). Overall, the cost per person was high in year 1 compared with years 2 through year 5 for each component.

The opinions expressed by authors contributing to this journal do not necessarily reflect the opinions of the U.S. Department of Health and Human Services, the Public Health Service, the Centers for Disease Control and Prevention, or the authors' affiliated institutions. 


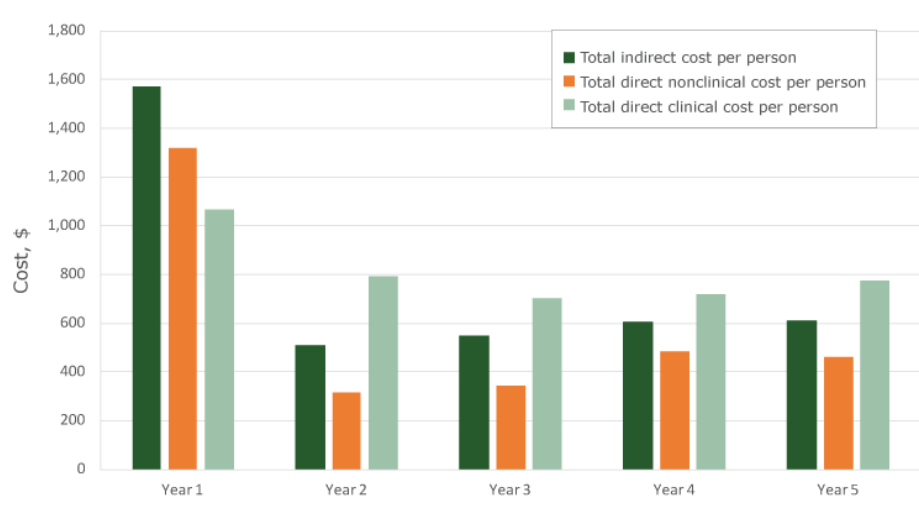

Figure. Five-year trends, cost per person screened, Colorectal Cancer Control Program, calculated on the basis of 124 program years, 2009-2014.

Cost per person by type of screening test varied significantly across the 3 test types (Table 3 ). On average, screening tests cost $\$ 2,060$ per person, ranging from $\$ 1,057$ for both tests to $\$ 3,153$ for colonoscopy. All components were, on average, most expensive for colonoscopy programs; total costs per person were $\$ 1,369$ for direct clinical costs, $\$ 863$ for nonclinical costs, and $\$ 921$ for indirect costs. By comparison, total cost per person for FOBT/FIT were $\$ 280$ for direct clinical costs, \$375 for direct nonclinical costs, and $\$ 636$ for indirect costs. Total per person costs for both tests were $\$ 411$ for direct clinical costs, $\$ 173$ for direct nonclinical cost was, and $\$ 473$ for indirect cost.

Examining the estimates for adjusted total cost per person, we found that programs using colonoscopy screening had an average $\$ 1,104$ higher total cost per person served compared with programs using FOBT/FIT tests in years 2 through 5 (Table 4). Increased size of the population served lowered total cost significantly; in years 2 through 5, average costs for programs with medium populations were $\$ 899$ lower than programs with small populations served, and programs with large populations were $\$ 1,313$ lower.

The total number of people screened had some effect on the direct clinical cost per person; programs with large populations screened had \$292 lower costs than programs with small populations screened. Colonoscopy programs had a higher direct clinical cost than FOBT/FIT programs (\$2,365 higher).

Our estimates for total direct nonclinical cost per person served show that type of screening test did not affect direct nonclinical costs (Table 4). Similar to total costs, costs for programs with large populations served were $\$ 352$ lower than programs with small populations served, whereas costs for programs with medium populations served were \$270 lower.
We also found that total indirect cost per person served was significantly lower among programs with larger populations served (Table 4). The average indirect cost per person served was $\$ 467$ lower among programs with a large population served and \$320 lower among programs with a medium population served, compared with programs with a small population served. Region also significantly affected these costs. Programs in the Northeast had an average $\$ 179$ lower indirect cost per person served than programs in the South.

\section{Discussion}

We compared the clinical and nonclinical costs across program years among CRCCP grantees offering colonoscopy, FOBT/FIT, or both tests for CRC screening. Our findings expand on our prior analysis and use 5 years of data to quantify the presence of economies of scale - programs that screen a larger number of people had lower cost per person than programs that screen a smaller number of people. After controlling for type of screening test, programs serving large and medium-size populations had per-person costs that were about $\$ 1,300$ and $\$ 900$ lower, respectively, than programs serving small populations.

Another key finding from our study was that public health-led CRCCP programs incurred substantial nonclinical costs. These costs are important to consider when planning future programs. On average, these costs were lower for programs with large patient volumes than for programs with small patient volumes. These findings indicate that substantial fixed costs are associated with nonclinical activities. These results are further evidence that economies of scale exist in CRC screening programs, as reported in other studies (2-4).

Analysis of patterns in cost per person indicated differences in cost between the first year and subsequent years of the program. The average cost per person served in the first year was twice that of the other years. This higher cost in the first year likely reflects start-up costs incurred by the programs while planning and beginning implementation. Furthermore, the number of people screened was generally lower in the first year. Any nonclinical costs incurred in the first year would have to be distributed across a much smaller cohort. High start-up costs in the initial years of the program were also reported in other studies $(3,16,17)$, suggesting that first-year costs should perhaps be analyzed separately and not pooled with costs incurred in subsequent program years.

Additionally, we identified some differences across programs related to type of screening test used. The clinical cost of colonoscopy was almost 5 times the cost of FOBT/FIT per person when screening and diagnostic follow-up tests were included. Therefore, programs that use colonoscopy will only be able to screen about

\footnotetext{
The opinions expressed by authors contributing to this journal do not necessarily reflect the opinions of the U.S. Department of Health and Human Services, the Public Health Service, the Centers for Disease Control and Prevention, or the authors' affiliated institutions.
} 
one-fifth the number of people that FOBT/FIT programs can for the same level of funding in the initial years of the program. This cost would only affect the number of people screened in the short term because colonoscopy is recommended every 10 years for those at average risk and with normal results, whereas FOBT/FIT is recommended to be performed annually. The clinical costs over a 10-year period for colonoscopy and FOBT/FIT may not be substantially different. We did not find any consistent evidence of variation in indirect costs and direct nonclinical costs by type of screening test used. FOBT/FIT tests were the preferred approach when the primary goal was to offer first-time screening to a large cohort over a short period; we did not study FOBT/FIT with repeated testing. Future studies could assess additional program costs that may be incurred, to ensure adherence with colorectal cancer screening recommendations over the long term. Furthermore, we found some regional and screening test-related differences in indirect costs; future studies could explore whether these findings are replicated in other settings and the possible reasons for these differences.

The strength of the present cost analysis is that we were able to perform high-quality analysis by collecting and quantifying resources and using consistent definitions for program activities. Furthermore, we collected data across 5 years from multiple programs to yield a substantial panel data set of 124 program years. These cost data were consistently collected over a longer period than any other federally supported screening program and allowed for multivariate analysis, controlling for some determinants of potential variation across the programs.

Our analysis has several potential limitations. First, we used program year to assess potential year-to-year variation, but programs generally operate on a continuous basis. Therefore, screening tests could be performed in one year, while diagnostic follow-up and treatment, if required, could be provided in the following year. As a result, classification of costs and number screened in specific periods are not always an accurate reflection of program activities. Second, the study does not account for cost per patient over an extended period to compare the long-term cost of colonoscopy versus FOBT/FIT-based programs. We only report cost for the first testing period (screening and diagnostic tests required), and our estimates do not provide the overall cost of FIT/FOBT and colonoscopy programs. Third, there could be variation across programs by type of screening test used (eg, colonoscopy vs FOBT/ FIT). This variation could influence the costs reported and may not have been adequately controlled in our analysis. Future research could systematically assess the factors that can lead to cost differences of activities by type of screening test selected.
Our analysis of the activity-based cost data across 5 years of the CRCCP reveals potential economies of scale: programs with larger screening volume incurred a lower cost per person served than smaller-volume programs. Therefore, encouraging partnerships to foster large-scale programs could be more efficient than funding multiple small screening programs. Additionally, CRC screening programs incur substantial nonclinical costs, regardless of type of test the program offers. Future CRC control programs might consider both these clinical and nonclinical costs when planning program implementation and evaluating program cost-effectiveness.

\section{Acknowledgments}

Funding support for Sujha Subramanian, Sonja Hoover, and Maggie Cole-Beebe was provided by the CDC (contract no. 200-200827958, Task order 01) to RTI International. The provision of data by grantees was supported through funding under a cooperative agreement with CDC. No copyrighted material, surveys, instruments, or tools were used in this manuscript. The findings and conclusions in this article are those of the authors and do not necessarily represent the official position of CDC.

\section{Author Information}

Corresponding Author: Sujha Subramanian, PhD, RTI International, 307 Waverley Oaks Rd, Ste 101, Waltham, MA 02452. Telephone: 781-788-8100. Email: ssubramanian@rti.org.

Author Affiliations: ${ }^{1}$ RTI International, Waltham, Massachusetts. ${ }^{2}$ Centers for Disease Control and Prevention, Atlanta, Georgia.

\section{References}

1. Subramanian S, Tangka FKL, Hoover S, Royalty J, DeGroff A, Joseph D. Costs of colorectal cancer screening provision in CDC's Colorectal Cancer Control Program: comparisons of colonoscopy and FOBT/FIT based screening. Eval Program Plann 2017;62:73-80.

2. Subramanian S, Ekwueme D, Gardner J, Kramer C, Bapat BS, Tangka F. Identifying and controlling for program-level differences in comparative cost analysis: lessons from the economic evaluation of the National Breast and Cervical Cancer Early Detection Program. Eval Program Plann 2008; 31(2):136-44.

3. Ekwueme DU, Gardner JG, Subramanian S, Tangka FK, Bapat B, Richardson LC. Cost analysis of the National Breast and Cervical Cancer Early Detection Program - selected states 2003 to 2004. Cancer 2008;112(3):626-35. 
4. Trogdon JG, Ekwueme DU, Subramanian S, Crouse W. Economies of scale in federally-funded state-organized public health programs: results from the National Breast and Cervical Cancer Early Detection Programs. Health Care Manage Sci 2014;17(4):321-30.

5. Anderson DW, Bowland BJ, Cartwright WS, Bassin G. Service-level costing of drug abuse treatment. J Subst Abuse Treat 1998;15(3):201-11.

6. Drummond M, Schulpher M, Torrance G, O’Brien B, Stoddard G. Methods for the economic evaluation of health care programmes. Oxford (UK): Oxford University Publishing; 2005.

7. French MT, Dunlap LJ, Zarkin GA, McGeary KA, McLellan AT. A structured instrument for estimating the economic cost of drug abuse treatment. The Drug Abuse Treatment Cost Analysis Program (DATCAP). J Subst Abuse Treat 1997; 14(5):445-55.

8. Salomé HJ, French MT, Miller M, McLellan AT. Estimating the client costs of addiction treatment: first findings from the client drug abuse treatment cost analysis program (Client DATCAP). Drug Alcohol Depend 2003;71(2):195-206.

9. Subramanian S, Ekwueme DU, Gardner JG, Trogdon J. Developing and testing a cost-assessment tool for cancer screening programs. Am J Prev Med 2009;37(3):242-7.

10. Hoover S, Subramanian S, Tangka F. Developing a WebBased Cost Assessment Tool for Colorectal Cancer Screening Programs. Prev Chronic Dis 2019;16:180336.

11. Tangka F, Subramanian S, Hoover S, Cole-Beebe M, DeGroff A, Joseph D, et al. Cost and factors associated with expenditures on screening promotion activities in CDC's Colorectal Cancer Control Program. Prev Chronic Dis 2019; 16:180337. . Forthcoming.

12. Liu L, Strawderman RL, Cowen ME, Shih Y-CT. A flexible two-part random effects model for correlated medical costs. J Health Econ 2010;29(1):110-23.

13. Manning WG, Basu A, Mullahy J. Generalized modeling approaches to risk adjustment of skewed outcomes data. J Health Econ 2005;24(3):465-88.

14. McCulloch CE. Maximum likelihood algorithms for generalized linear mixed models. J Am Stat Assoc 1997; 92(437):162-70.

15. Griswold M, Parmigiani E, Potoksky A, Lipscomb J. Analyzing health care costs: a comparison of statistical methods motivated by Medicare colorectal cancer charges. Biostatistics 2004;1(1):1-23.

16. Tangka FK, Subramanian S, Bapat B, Seeff LC, DeGroff A, Gardner J, et al. Cost of starting colorectal cancer screening programs: results from five federally funded demonstration programs. Prev Chronic Dis 2008;5(2):A47.
17. Subramanian S, Tangka FK, Hoover S, Degroff A, Royalty J, Seeff LC. Clinical and programmatic costs of implementing colorectal cancer screening: evaluation of five programs. Eval Program Plann 2011;34(2):147-53.

\footnotetext{
The opinions expressed by authors contributing to this journal do not necessarily reflect the opinions of the U.S. Department of Health and Human Services, the Public Health Service, the Centers for Disease Control and Prevention, or the authors' affiliated institutions.
} 


\section{Tables}

Table 1. Program Characteristics and Clinical Services by Type of Primary Screening Test for All Program Years ${ }^{\mathrm{a}}$, Centers for Disease Control and Prevention Colorectal Cancer Control Program, 2009-2014

\begin{tabular}{|c|c|c|c|c|}
\hline \multirow[b]{2}{*}{ Characteristic } & \multirow[b]{2}{*}{ All $(N=124)$} & \multicolumn{3}{|c|}{ By Type of Test } \\
\hline & & Colonoscopy $(n=55)$ & FOBT/FIT ( $n=39)$ & $\begin{array}{l}\text { Colonoscopy and FOBT/FIT } \\
\qquad(n=30)\end{array}$ \\
\hline By screening test & $\mathrm{NA}$ & 44.4 & 31.5 & 24.2 \\
\hline \multicolumn{5}{|c|}{ Region, mean (95\% confidence interval) } \\
\hline Northeast $^{\mathrm{b}}$ & $20.2(13.0-27.3)$ & $36.4(23.2-49.5)$ & 0 & $16.7(2.5-30.8)$ \\
\hline Midwest $^{\mathrm{c}}$ & $18.6(11.6-25.5)$ & $9.1(1.3-16.9)$ & $28.2(13.4-43.0)$ & $23.3(7.3-39.4)$ \\
\hline South & $17.7(10.9-24.6)$ & $23.6(12.1-35.2)$ & $10.3(0.3-20.2)$ & $16.7(2.5-30.8)$ \\
\hline West $^{\mathrm{c}}$ & $43.6(34.7-52.4)$ & $30.9(18.3-43.5)$ & $61.5(45.6-77.5)$ & $43.3(24.5-62.2)$ \\
\hline \multicolumn{5}{|c|}{ Size of population screened by program size ${ }^{d}$, mean $(95 \%$ confidence interval) } \\
\hline Large population $^{\mathrm{b}}$ & $34.7(26.2-43.2)$ & $10.9(2.4-19.4)$ & $46.2(29.8-62.5)$ & $63.3(45.0-81.6)$ \\
\hline Medium population $^{c}$ & $36.3(27.7-44.9)$ & $45.5(31.9-59.0)$ & $38.5(22.5-54.4)$ & $16.7(2.5-30.8)$ \\
\hline Small population ${ }^{b}$ & $29.03(20.93-37.13)$ & $43.6(30.1-57.2)$ & $15.4(3.5-27.2)$ & $20.0(4.8-35.2)$ \\
\hline \multicolumn{5}{|c|}{ Program reach, mean (95\% confidence interval) } \\
\hline No. of people screened ${ }^{b}$ & $848.0(540.8-1,155.3)$ & $253.9(208.6-299.2)$ & $683.3(518.5-848.1)$ & $2151.5(981.6-3321.4)$ \\
\hline No. of people under surveillance ${ }^{b}$ & $23.5(15.8-31.2)$ & $15.7(9.3-22.0)$ & $21.3(11.9-30.8)$ & $40.8(13.4-68.1)$ \\
\hline No. of diagnostic tests performed ${ }^{b}$ & $41.3(25.1-57.5)$ & $7.3(5.2-9.4)$ & $44.2(29.9-58.5)$ & 99.9 (38.7-161.0) \\
\hline No. of polyps detected ${ }^{b}$ & $47.5(41.0-54.1)$ & $61.4(50.7-72.0)$ & $27.2(20.0-34.4)$ & $48.6(35.1-62.1)$ \\
\hline
\end{tabular}

Abbreviation: FOBT/FIT, fecal occult blood test/fecal immunochemical test; NA, not applicable.

${ }^{a}$ Unit of analysis is program year. Total sample size available for analysis was 124 program years over the 5 -year period. We used the $x^{2}$ test to test for differences across the types of colorectal cancer screening tests.

${ }^{\mathrm{b}} P<.001$.

${ }^{\mathrm{c}} P<.05$.

${ }^{d}$ Small population $=228,339-736,635 ;$ medium population $=854,624-1,618,255$; large population $=1,749,719-9,472,316$. 
Table 2. Program Testing Method by Program Characteristics, Centers for Disease Control and Prevention Colorectal Cancer Control Program, 2009-2014

\begin{tabular}{|c|c|c|c|}
\hline Characteristic & Colonoscopy & FOBT/FIT & Colonoscopy and FOBT/FIT \\
\hline \multicolumn{4}{|l|}{ Region } \\
\hline Northeast $(n=20)$ & $80.0(60.8$ to 99.2$)$ & 0 & 20.0 (7.9 to 39.2) \\
\hline Midwest $(n=20)$ & 20.0 (0.8 to 39.2) & 50.0 (6.0 to 74.0$)$ & 30.0 (8.0 to 52.0$)$ \\
\hline South $(n=19)$ & 57.9 (33.5 to 82.3) & 21.15 (0.9 to 41.2$)$ & 21.1 (0.9 to 41.2$)$ \\
\hline West $(n=46)$ & 30.4 (16.6 to 44.3$)$ & 47.8 (32.8 to 62.8$)$ & $21.7(9.4$ to 34.1$)$ \\
\hline \multicolumn{4}{|l|}{ Population density } \\
\hline Large population $(n=38)$ & $13.2(1.9$ to 24.4$)$ & $42.1(25.7$ to 58.6$)$ & 44.7 (28.2 to 61.3 ) \\
\hline Medium population $(n=43)$ & NA & 34.9 (20.0 to 49.7$)$ & 9.3 (0.3 to 18.4$)$ \\
\hline Small population $(n=24)$ & 66.7 (46.3 to 87.0$)$ & 20.8 (3.3 to 38.4$)$ & 12.5 (-1.8 to 26.8$)$ \\
\hline
\end{tabular}

Abbreviation: FOBT/FIT, fecal occult blood test/fecal immunochemical test; NA, not applicable.

${ }^{\text {a }}$ Values are percentage (95\% confidence interval). 
Table 3. Cost per Person Screened by Type of Primary Test, Centers for Disease Control and Prevention Colorectal Cancer Control Program, 2009-2014

\begin{tabular}{|c|c|c|c|c|}
\hline \multirow[b]{2}{*}{ Type of Cost ${ }^{a}$} & \multirow[b]{2}{*}{ All } & \multicolumn{3}{|c|}{ By Type of Test } \\
\hline & & Colonoscopy & FOBT/FIT & $\begin{array}{c}\text { Colonoscopy and FOBT/ } \\
\text { FIT }\end{array}$ \\
\hline Total cost per person $^{\mathrm{b}}$ & $2,060(1,565-2,556)$ & $3,153(2,175-4,132)$ & $1,291(787-1,794)$ & $1,057(631-1,482)$ \\
\hline Total direct clinical cost per person ${ }^{b}$ & $795(631-958)$ & $1,369(1,069-1,669)$ & $280(216-343)$ & $411(283-539)$ \\
\hline Total direct nonclinical cost per person ${ }^{b}$ & $543(260-826)$ & $863(261-1,465)$ & $375(87-663)$ & $173(50-295)$ \\
\hline Total indirect cost per person ${ }^{\mathrm{b}}$ & $723(535-912)$ & $921(552-1,290)$ & $636(390-882)$ & $473(238-708)$ \\
\hline
\end{tabular}

Abbreviation: FOBT/FIT, fecal occult blood test/fecal immunochemical test.

${ }^{\text {a }}$ All costs include in-kind contributions and were adjusted by using the Employment Cost Index for regional differences. Values are US dollars (95\% confidence interval).

${ }^{\mathrm{b}} P<.001$. We used the $\mathrm{X}^{2}$ test to test for differences across the types of CRC screening tests. 
Table 4. Adjusted Cost per Person Screened, Years 2 to 5, Centers for Disease Control and Prevention Colorectal Cancer Control Program, 2009-2014 ${ }^{a}$

\begin{tabular}{|c|c|c|c|c|}
\hline Variable & Total Per Person & Direct Clinical & Direct Nonclinical & Indirect \\
\hline \multicolumn{5}{|l|}{ Region } \\
\hline South & & & & 1 [Reference] \\
\hline Northeast & (95) (-550 to 537$)$ & (122) $(-355$ to 251$)$ & $70(-177$ to 587$)$ & $(179)^{b}(-301$ to -2$)$ \\
\hline Midwest & (31) $(-513$ to 642$)$ & $28(-274$ to 524$)$ & (6) $(-218$ to 447$)$ & (76) (-231 to 150$)$ \\
\hline West & $318(-179$ to 976$)$ & (16) $(-254$ to 337$)$ & $222(-77$ to 797$)$ & 90 (-89 to 336$)$ \\
\hline \multicolumn{5}{|c|}{ Size of population served by the program ${ }^{c}$} \\
\hline Small population served & & & & 1 [Reference] \\
\hline Large population served & $-1,313^{\mathrm{C}}(-1,412$ to -1181$)$ & $-292^{b}(-445$ to -62$)$ & $-352^{d}(-377$ to -302$)$ & $-467^{d}(-495$ to -429$)$ \\
\hline Medium population served & $-899^{d}(-1,098$ to -636$)$ & $-118(-325$ to 192$)$ & $-270^{d}(-333$ to -150$)$ & $-320^{d}(-388$ to -226$)$ \\
\hline \multicolumn{5}{|l|}{ Screening test } \\
\hline FOBT/FIT & & & & 1 [Reference] \\
\hline Colonoscopy & $1,104^{d}$ (439 to 1,974$)$ & $\begin{array}{r}2,365^{\mathrm{c}} \text { (1 to } 319 \text { to } 3 \text { to } \\
940)\end{array}$ & $76(-139$ to 469$)$ & $-64(-196$ to 115$)$ \\
\hline FOBT/FIT and colonoscopy & $-215(-563$ to 237$)$ & $249(-49$ to 675$)$ & $-108(-245$ to 151$)$ & $-139(-252$ to 15$)$ \\
\hline
\end{tabular}

Abbreviation: FOBT/FIT, fecal occult blood test/fecal immunochemical test.

${ }^{a}$ All costs include in-kind contributions and were adjusted by using the Employment Cost Index for regional differences. All estimates are based on multivariate analysis; each column is a separate regression. Values are dollars (95\% confidence interval). Results are for years $2-5(\mathrm{~N}=105)$.

${ }^{\mathrm{b}} P<.05$.

${ }^{\mathrm{c}}$ Small population $=<235$; medium population $=235-500$; large population $=>500$.

${ }^{\mathrm{d}} P<.001$.

The opinions expressed by authors contributing to this journal do not necessarily reflect the opinions of the U.S. Department of Health and Human Services, the Public Health Service, the Centers for Disease Control and Prevention, or the authors' affiliated institutions. 